\title{
Parameterization of vertical dispersion coefficient over idealized rough surfaces in isothermal conditions
}

\author{
Chun-Ho Liu*+ ${ }^{*}$, Ziwei Mo ${ }^{\dagger}$ and Zhangquan $\mathrm{Wu}^{\dagger}$
}

\begin{abstract}
Urban air quality is an important problem nowadays because of the close proximity of sources and receptors in densely built environment. Gaussian plume models have been commonly employed in the industry to estimate air pollution impact over open terrain for decades. However, they should be applied cautiously to urban environment in view of the complicated recirculating flows and turbulence-generation mechanism in the wakes around/over buildings. In particular, one of the key components in Gaussian plume models, dispersion coefficient $\sigma_{z}$, is usually determined empirically based on atmospheric stratification that might overlook the effect of rough urban surfaces in the bottom of atmospheric boundary layer, resulting in prediction uncertainty. In this paper, we report our recent study of the transport processes over idealized rough surfaces (repeated ribs in crossflows) to simulate the flows and transport processes after a ground-level pollutant source in crossflows over hypothetical urban areas. The effect of aerodynamic resistance (controlled by the rib separation $b$ ) on pollutant plume dispersion (measured by vertical dispersion coefficient $\sigma_{z}$ ) is critically examined. First of all, analytical solution shows that $\sigma_{z}$ is proportional to $x^{1 / 2} \times \delta^{1 / 2} \times f^{1 / 4}$, where $x$ is the downwind distance after the pollutant source, $\delta$ the turbulent boundary layer thickness, $f\left(=2 u_{\tau}^{2} / U_{2}^{2}\right.$ the friction factor, $u_{\tau}$ the friction velocity and $U_{\infty}$ the free-stream wind speed. Afterward, a complementary approach, using both wind-tunnel measurements and large-eddy simulation results, is used to verify the newly developed theoretical hypothesis. Although mild discrepancies are observed among various solutions (due to unavoidable scaling effect), the aforementioned analytical proportionality is clearly depicted. The findings unveil the weakness of conventional practice using Gaussian plume models, proposing a new parameterization of dispersion coefficient for pollutant plume dispersion over urban areas.
\end{abstract}

Keywords: Air pollution modeling, Impact on urban inhabitants

\section{Background}

Air pollution poses major threat to premature mortality (Lelieveld et al. 2015) but its levels over $80 \%$ of the cities in the world are unhealthy (WHO 2016). The impact is in fact worsening because of the rapidly growing population, number and size of cities (DESA 2016). Numerous parties are involved in urban development that implies the giant economic activities behind. Besides, air quality

\footnotetext{
*Correspondence: liuchunho@graduate.hku.hk

${ }^{\dagger}$ Chun-Ho Liu, Ziwei Mo and Zhangquan Wu contributed equally to this paper

Department of Mechanical Engineering, The University of Hong Kong,

7/F, Haking Wong Building, Pokfulam Road, Hong Kong, China
}

is a key factor affecting the living quality of public. An advanced understanding of how urban morphology influences air quality in neighborhood scales is, therefore, crucial nowadays. Apparently, there is a need for costeffective modeling solutions to estimate the transport of air pollutants in urban areas.

Among various mathematical models, the conventional Gaussian model (Roberts 1923) is most widely adopted to solve practical problems (Moreira et al. 2006) in particular for regulatory enactment (Briant et al. 2013), air toxic assessment (Scheffe et al. 2016) and continental pollutant transport (Tsuang et al. 2003). For urban applications, however, the results of Gaussian model must be interpreted with caution because of the complicated 
near-wall turbulent transport processes right over urban canopy layers (Britter and Hanna 2003). Generally, the drag induced by (rough) urban surfaces increases the aerodynamic resistance (Raupach et al. 1991; MacDonald et al. 1998) that in turn modifies the pollutant transport processes aloft. Whilst, most schemes of dispersion coefficient currently available were derived based on atmospheric stability but not urban morphology (Pasquill and Smith 1983; Turner 1994). Whereas, the aerodynamic effect of roughness elements is often more influential to the transport processes in the vicinity over urban areas.

From the engineering point of view, widening plume coverage over (rough) urban areas is attributed to the elevated turbulence kinetic energy (TKE) in response to enhanced ground-level aerodynamic resistance (Walcek 2002). Schemes have been updated to handle pollutant dispersion over dense buildings (Briggs 1973) such as modifying power-law wind profiles (Sharma and Myrup 1975) together with dispersion coefficients (Skupniewicz and Schacher 1986) to handle the pollutant distribution over urban areas. Whereas the empirical solution approach was rather site specific that weakened our fundamental understanding of the transport processes (Venkatram et al. 2005). In view of the importance to urban inhabitants and public health, extensive field measurements (Davidson et al. 1995; Mavroidis and Griffiths 2001), laboratory experiments (Gromke 2011; Chung et al. 2015) and mathematical modeling (Inagaki et al. 2012; Wong and Liu 2013) have been carried out to foster the theory of transport processes over rough surfaces. Whereas, the functional form of dispersion coefficients over urban areas is not yet formulated.

The authors have persisted to address the above questions for years. We extend our on-going research effort, by looking into the flows and tracer dispersion over hypothetical urban areas, to develop a fundamental understanding of the transport processes using concurrently wind-tunnel experiments and large-eddy simulation (LES). Utilizing the theoretical solution and data archive, we attempt to parameterize the functional form of dispersion coefficients over (hypothetical) urban areas. The conventional Gaussian-plume framework is used because of its solid theoretical basis. We simply use the aerodynamic resistance to model the turbulent transport processes rather than simulate the flows over complicated urban morphology in refined scales. Unlike computational fluid dynamics (CFD), dispersion parameterizations possess the benefit of quick and reasonably reliable solution at affordable costs. This paper records our development and verification of dispersion coefficients for urban setting. The first section introduces the problem background and reviews the available literature. Afterward, the theory of plume dispersion is derived analytically. Next, the methodology, both windtunnel experiments and LES, is detailed. The findings of the characteristic dynamics and pollutant transport processes related to urban rough surfaces are reported before the conclusions are drawn.

\section{Theoretical background}

Dispersion coefficient $\sigma_{z}$ in isothermal conditions is a function of atmospheric turbulence, surface roughness, mean-wind speed $U$ and the distance $x$ after the pollutant source. It is usually described by the classic $k$-theory model

$$
\sigma_{z}^{2}=2 k_{z} t=2 k_{z} \frac{x}{U}
$$

where $k_{z}$ is the eddy diffusivity in the vertical direction $z$ and $t(=x / U)$ the pollutant travel time from the source to the receptor in the streamwise direction $x$. We use the mixing-length theory to handle the turbulent transport processes so the eddy diffusivity is proportional to the characteristic length and velocity scales. For the nearwall dynamics over urban areas, we take the turbulent boundary layer (TBL) thickness $\delta$ and the friction velocity $u_{\tau}$ as the characteristic length and velocity scales, respectively. Hence,

$$
k_{z} \propto \delta \times u_{\tau} .
$$

Equation (1) is then simplified to

$$
\sigma_{z}^{2} \propto x \times \delta \times \frac{u_{\tau}}{U} .
$$

We substitute the friction factor $f\left(=2 u_{\tau}^{2} / U_{\infty}{ }^{2}\right)$ into Eq. (3) that arrives the basic functional form of dispersion coefficient

$$
\frac{\sigma_{z}}{h} \propto\left(\frac{x}{h}\right)^{1 / 2} \times\left(\frac{\delta}{h}\right)^{1 / 2} \times f^{1 / 4}
$$

in a dimensionless manner where $h$ is the size of roughness elements used for scaling.

\section{Methods}

\section{Wind tunnel experiments}

Experiments are conducted in the open-circuit wind tunnel in the Department of Mechanical Engineering, The University of Hong Kong in isothermal conditions (Fig. 1a). It is noted that atmospheric boundary layer (ABL) is seldom isothermal. The findings in this paper are only applicable to the bottom ABL right over buildings where the turbulence is largely generated by 

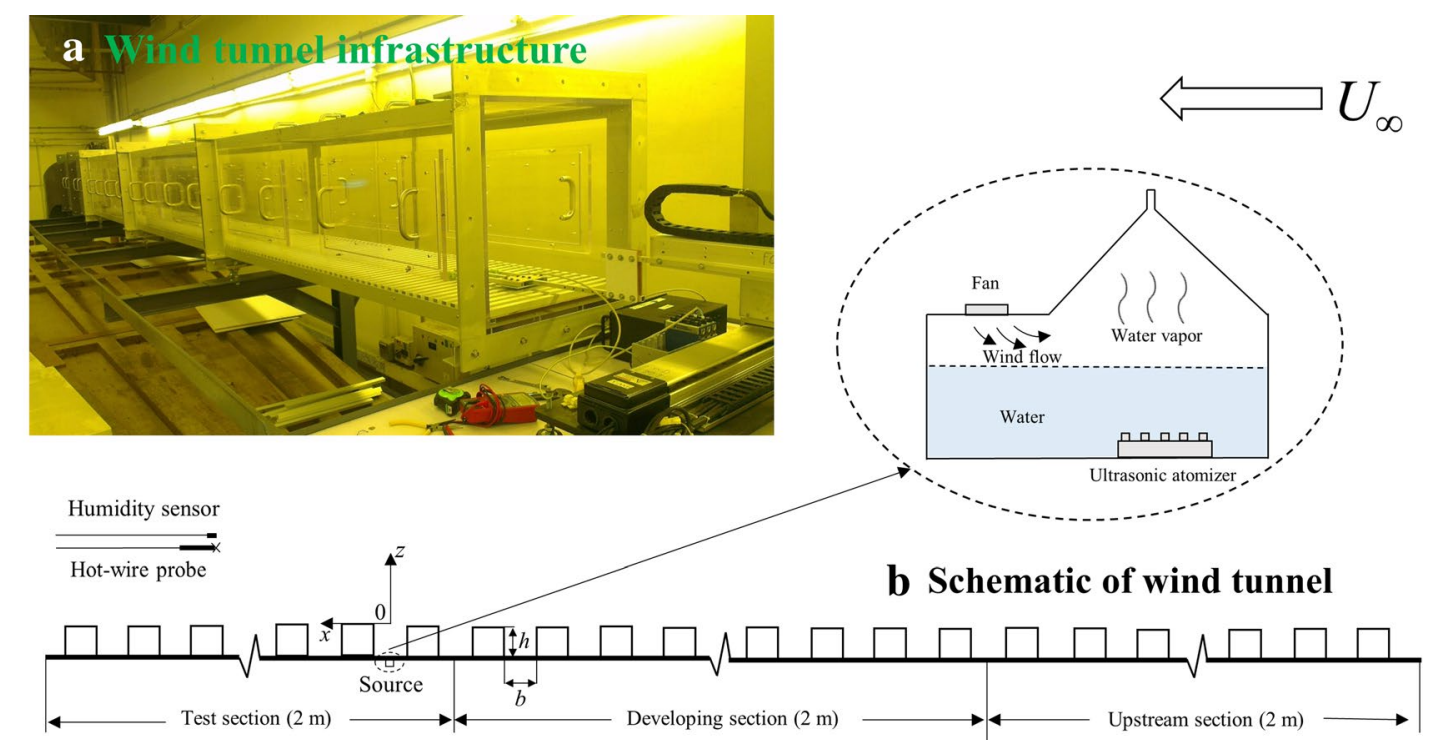

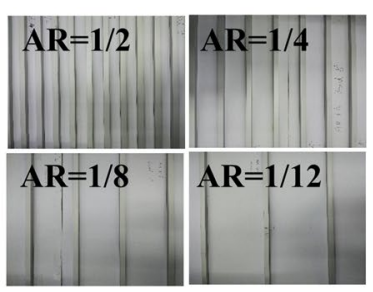

c Rib configuration

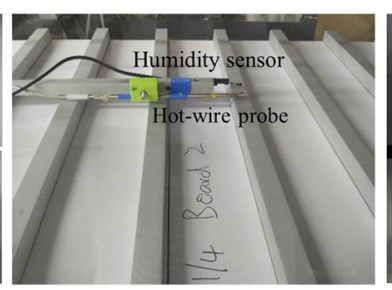

d Sensor location

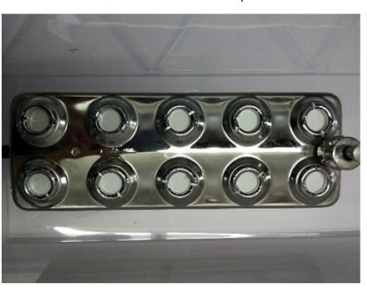

e $\mathrm{H}_{2} \mathrm{O}$ atomizer

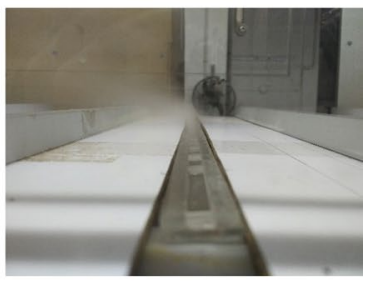

f Source location

Fig. 1 Experimental setup in the wind tunnel

mechanical wind shear rather than buoyancy. Moreover, the modeled TBL is shallow compared with the real $\mathrm{ABL}$, therefore, there is no major difference in vertical pressure in the current study. Hence, the results obtained from laboratory experiments and mathematical modeling are applicable to neutrally stratified ABL (zero potential temperature gradient). In addition to the flow straightener in-between the settling chamber and the contraction cone, a honeycomb filter is installed afterward to reduce the background turbulence intensity. The flows are driven by a three-phase, electric blower whose power is controlled by a frequency invertor. The acrylic-made wind-tunnel test section sizes $6000 \mathrm{~mm}$ (length) $\times 560 \mathrm{~mm}$ (width) $\times 560 \mathrm{~mm}$ (height). The roughness elements, which are used to model (hypothetical) urban areas, are glued on the testsection floor, developing the TBL. The design of wind speed is in the range of $1 \mathrm{~m} \mathrm{~s}^{-1} \leq U_{\infty} \leq 15 \mathrm{~m} \mathrm{~s}^{-1}$. The free-stream wind speed, which is kept in the range of $3 \mathrm{~m} \mathrm{~s}^{-1} \leq U_{\infty} \leq 7 \mathrm{~m} \mathrm{~s}^{-1}$, is also monitored by a pitot tube upstream of the test section (Fig. 1b). Details of our wind-tunnel infrastructure are available elsewhere (Mo and Liu 2017).
Two-dimensional (2D) roughness elements in the form of identical ribs in crossflows are used to model rough surfaces (Fig. 1c). Aluminum squares of size $h=19 \mathrm{~mm}$ are placed evenly apart on which the aerodynamic resistance is controlled by their separation $b$. Four values of separations are tested to fabricate idealized urban street canyons whose building-height-to-street-width (aspect) ratio, $A R$, is in the range of $1 / 12 \leq A R \leq 1 / 2$, covering the classic regimes of skimming flows, wake interference and isolated roughness (Oke 1988). The corresponding friction factor is in the range of $8 \times 10^{-3} \leq f \leq 13 \times 10^{-3}$ (Table 1). Moreover, two different levels of wind speeds are adopted to ensure Reynolds-number independence.

Flows are probed by constant-temperature (CT) hotwire anemometer (HWA) with a cross-wire design to measure streamwise $u$ and vertical $w$ velocity components (Fig. 1d). The sensing element of the probe consists of a pair of $5-\mu \mathrm{m}$ (diameter) platinum-plated tungsten wires. By copper electroplating, the active length of the sensing element is $2 \mathrm{~mm}$. The included angle between the two wires is $100^{\circ}$ that helps reduce the error due to inadequate yaw response in elevated turbulence intensity (Cheng and Castro 2002). The CT HWA probe is 
Table 1 Configuration of the flows over hypothetical urban areas in wind tunnel experiments and LES

\begin{tabular}{|c|c|c|c|c|c|c|c|c|c|c|c|c|c|}
\hline & \multicolumn{8}{|c|}{ Wind tunnel experiments } & \multicolumn{5}{|l|}{ LES } \\
\hline & W-AL & W-AH & W-BL & W-BH & $\mathrm{W}-\mathrm{CL}$ & $\mathrm{W}-\mathrm{CH}$ & W-DL & W-DH & M-A & M-B & $M-C$ & M-D & $M-E$ \\
\hline $\operatorname{AR}(=h / b)$ & $1 / 2$ & & $1 / 4$ & & $1 / 8$ & & $1 / 12$ & & 1 & $1 / 2$ & $1 / 3$ & $1 / 5$ & $1 / 11$ \\
\hline \multicolumn{14}{|l|}{$\operatorname{Rib}\left[\times 10^{3} \mathrm{~m}\right]$} \\
\hline$h$ & 19 & & & & & & & & & & 1 & & \\
\hline$b$ & 38 & & 76 & & 152 & & 228 & & 1 & 2 & 3 & 5 & 11 \\
\hline$\delta[\mathrm{m}]$ & 0.240 & 0.245 & 0.260 & 0.265 & 0.285 & & 0.265 & 0.260 & & & 11 & & \\
\hline$\delta / h$ & 12.6 & 12.9 & 13.7 & 14.0 & 15.0 & & 14.0 & 13.7 & & & 11 & & \\
\hline$U_{\infty}\left[\mathrm{m} \mathrm{s}^{-1}\right]$ & 3.28 & 6.66 & 3.31 & 6.61 & 3.28 & 6.70 & 3.29 & 6.60 & 1.01 & 0.81 & 0.65 & 0.58 & 0.56 \\
\hline$U_{m}\left[\mathrm{~m} \mathrm{~s}^{-1}\right]$ & 2.92 & 5.91 & 2.99 & 5.95 & 2.84 & 5.88 & 2.91 & 5.85 & 0.86 & 0.66 & 0.51 & 0.43 & 0.41 \\
\hline$u_{\tau}\left[\mathrm{m} \mathrm{s}^{-1}\right]$ & 0.18 & 0.38 & 0.22 & 0.45 & 0.22 & 0.47 & 0.22 & 0.47 & & & 0.047 & & \\
\hline$u_{\tau} / U_{\infty}\left[\times 10^{-3}\right]$ & 56 & 57 & 65 & 68 & 68 & 71 & 68 & 71 & 0.046 & 0.058 & 0.072 & 0.081 & 0.085 \\
\hline$u_{\tau} / U_{m}\left[\times 10^{-3}\right]$ & 63 & 65 & 72 & 76 & 78 & 81 & 77 & 80 & 0.055 & 0.071 & 0.092 & 0.11 & 0.11 \\
\hline$f\left[\times 10^{-3}\right]\left(=2 u_{T}^{2} / U_{\infty}^{2}\right.$ & 8.0 & 8.3 & 10.4 & 11.4 & 12.2 & 13.0 & 11.9 & 12.8 & 4.3 & 6.7 & 10.3 & 13.2 & 14.3 \\
\hline $\operatorname{Re}_{\delta}\left[\times 10^{3}\right]\left(=U_{\infty} \delta / v\right)$ & 79 & 163 & 86 & 175 & 93 & 191 & 87 & 172 & 111 & 89 & 72 & 63 & 61 \\
\hline $\operatorname{Re}_{h}\left[\times 10^{3}\right]\left(=U_{\infty} h / v\right)$ & 6 & 13 & 6 & 13 & 6 & 13 & 6 & 13 & 10 & 8 & 7 & 6 & 6 \\
\hline $\operatorname{Re}_{m}\left[\times 10^{3}\right]\left(=U_{m} h / v\right)$ & 6 & 11 & 6 & 11 & 5 & 11 & 6 & 11 & 9 & 7 & 5 & 4 & 4 \\
\hline $\operatorname{Re}_{T}\left(=u_{T} h / v\right)$ & 350 & 726 & 409 & 854 & 422 & 900 & 426 & 889 & & & 469 & & \\
\hline
\end{tabular}

positioned by a mechanical traversing system which is controlled by the National Instruments (NI) motion control unit (spatial resolution of $1 \mathrm{~mm}$ ). The analog CT HWA signal is digitalized by a 24-bit NI data acquisition module (NI 9239; offset error $\pm 0.05 \%$ for analog input $\pm 10.52 \mathrm{~V}$ ) mounted in a NI CompactDAQ classis (NI cDAQ-9188). The NI units are connected to a digital computer via a local area network (LAN) cable and the data sampling is managed by LabVIEW software. The sampling frequency is $2 \mathrm{kHz}$ and the sample size at each point is equal to $2^{17}$ that are sufficient for repeatability of mean and fluctuating signals. The CT HWA calibration is based on the universal calibration law of the Institute of Sound and Vibration (ISVR; Bruun 1971). Its readings are also compared with those of the pitot tube installed upstream during the experiments.

Water vapor is used as the tracer in the wind tunnel experiments. It is generated by an atomizer in the water tank (Fig. 1e) under the wind tunnel and is emitted via the ground-level line source in crossflows (Fig. 1f). An axial fan is used to drive the water vapor from the water tank to the line source. The flow speed induced by the fan is small (around $0.2 \mathrm{~m} \mathrm{~s}^{-1}$ ) that would not affect too much the core flows in the wind-tunnel test section. The water consumption rate for a set of experiment is about $2 \mathrm{~L}$ an hour. The water vapor is measured by Humidity and Temperature Sensor SHT 75 (SENSIRION AG Switzerland). Another SHT 75 is placed upstream to monitor the background humidity. The sensor response time is $8 \mathrm{~s}$ whose accuracy is $\pm 1.8 \%$. The tracer and flow measurements are collected separately to avoid inaccurate temperature measurements contaminated by water moisture.

\section{Large-Eddy simulation}

LES of the open-source CFD code Open-FOAM 4.1 (OpenFOAM 2018) is used in this paper. The atmospheric flows are assumed to be isothermal and incompressible that are calculated by the filtered continuity and Navier-Stokes equations. The LES TBL is shallow, therefore, incompressible flows are assumed. The (anisotropic part of) subgrid-scale (SGS) Reynolds stress is modeled by the Smagorinsky model (Smagorinsky 1963) together with the one-equation SGS model (Schumann 1975) to handle the SGS TKE conservation. The tracer transport is calculated by the advection-diffusion equation with the SGS eddy-viscosity model. The governing equations and numerical methods are reported elsewhere (Wu and Liu 2017)

The hypothetical urban areas consist of a number of idealized urban street canyons fabricated by identical square ribs of size $h$ (Fig. 2). The spatial domain sizes $72 h$ (length; approximately, depending on the separation among roughness elements $b) \times 12 h$ (width) $\times 12 h$ (height) that is composed of different number of street canyons depending on the AR. The core flows in the urban TBL are driven by the (background) pressure gradient $\Delta P_{x}$ normal to the street axes that represents the worst scenario in term of pollutant removal from street canyons. The domain extent is $12 h$ in the homogeneous 


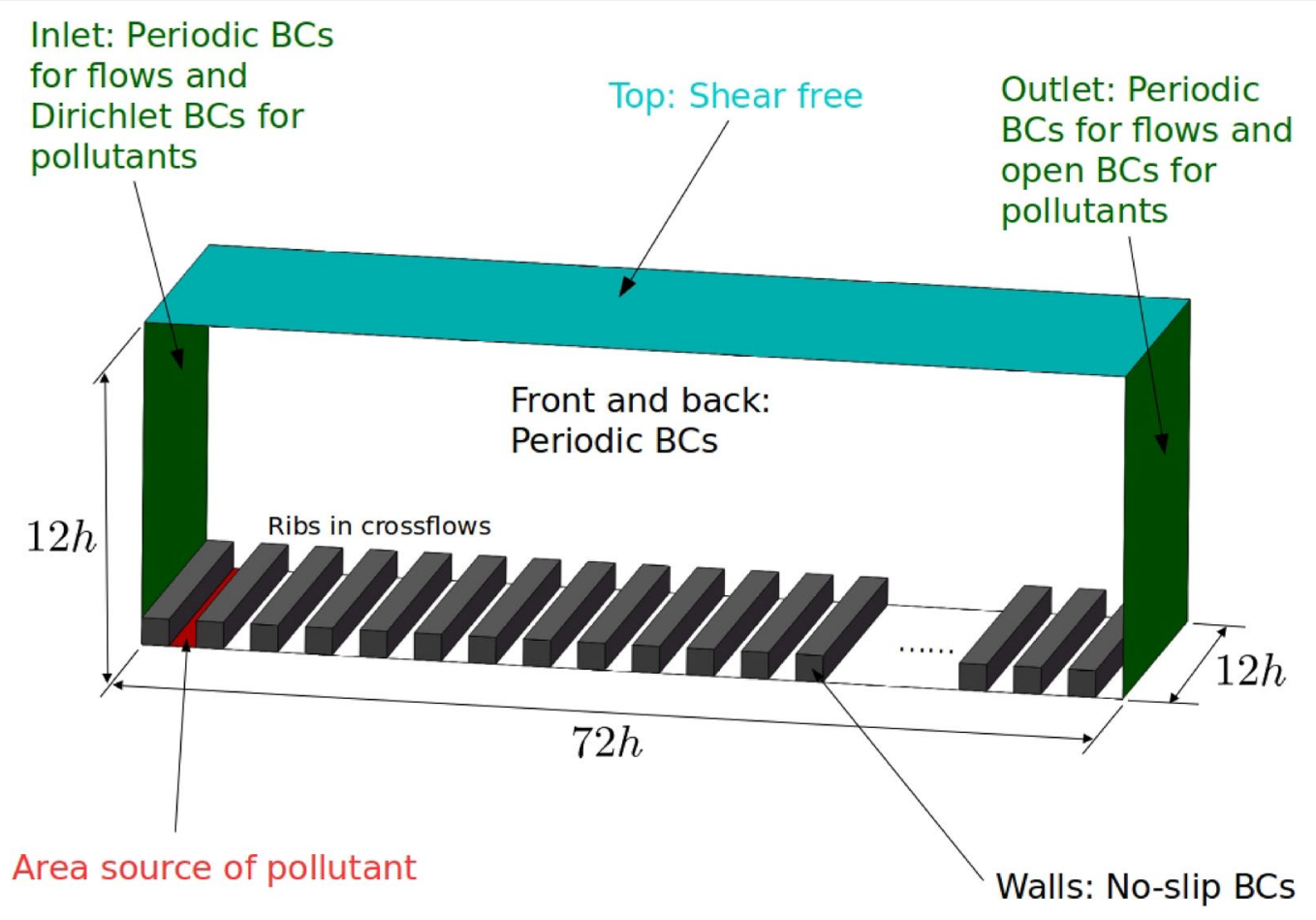

Fig. 2 LES computational domain and boundary conditions

spanwise $y$ direction. The infinitely long horizontal extent, in both streamwise $x$ and spanwise $y$ directions, is constructed by cyclic boundary conditions (BCs). Noslip BCs are applied on all the solid boundaries and a shear-free $\mathrm{BC}$ along the domain top. The prevailing wind enters the spatial domain from the upstream inflow with tracer-free fresh air. An area tracer source with constant concentration is placed on the ground level of the first street canyon from which a passive, inert tracer is continuously emitted into the computational domain. All solid boundaries are free of tracer on which Neumann BCs are applied. An open BC is prescribed at the downstream outflow, therefore, all the tracers are removed from the computational domain without reflection.

Because of geometric homogeneity, the size of mesh discretizing the spatial domain is uniform in the spanwise $y$ direction. Each street canyon comprises $N_{x} \times N_{y} \times N_{z}$ cells which are refined toward the solid boundaries in the $x$ and $z$ direction for improved accuracy. The urban TBL core over the array of street canyons is discretized into $L_{x} \times N_{y} \times L_{z}$ whose mesh size stretches to a factor of 3 in the vertical direction toward the domain top. The entire computational domain is thus composed of over $6 \times 10^{6}$ (hexahedral) cells whose size is in the order of $0.1 \mathrm{~h}$.

Finite volume method (FVM) is used to solve the governing equations. The implicit first-order-accurate backward differencing is used in the temporal domain in which the time increment is set to $0.01 \mathrm{~s}$. The gradient, divergence and Laplacian terms are calculated by the second-order-accurate Gaussian FVM integration based on the summation on cell faces. The pressure-velocity coupling in incompressible flows is handled by the combination of pressure-implicit with splitting of operators (PISO) and semi-implicit method for pressure-linked equations (SIMPLE). The symmetric and asymmetric equations systems are solved by the pre-conditioned conjugate gradient (PCG) and the pre-conditioned biconjugate gradient (PBiCG) methods, respectively. The residual of the iterative solvers is less than $10^{-8}$ for converged solution.

\section{Results}

The flow parameters in the wind tunnel experiments and LES are tabulated in Table 1 . The TBL thickness $\delta$ in the LES is the same as the domain height. In the wind tunnel experiments, on the other hand, it is taken at the height where the mean-wind speed $\langle\bar{u}\rangle$ converges to $99 \%$ of the free-stream one $U_{\infty}$. In this study, the function of TBLs is analogously to that of the mixed boundary layers in ABL. Thermal stratification is another factor in ABL development. While isothermal conditions are assumed, the TBLs are mainly affected by the bottom rough surfaces together with the mechanically generated turbulence. The buffer region upstream the test section is sufficiently 
long for flow development so the effect of inflow conditions is negligible in the current wind tunnel experiments. The TBL thickness $\delta$ over different configurations of roughness elements in the wind tunnel experiments is ranged from 12.6 to $15 h$ while it is equal to $11 h$ throughout the LESs (because of the domain size). The flows in the wind tunnel are driven by the blower upstream whose free-stream wind speed and friction velocity are in the range of $3.28 \mathrm{~m} \mathrm{~s}^{-1} \leq U_{\infty} \leq 6.7 \mathrm{~m} \mathrm{~s}^{-1}$ and $0.18 \mathrm{~m} \mathrm{~s}^{-1}$ $\leq u_{\tau} \leq 0.47 \mathrm{~m} \mathrm{~s}^{-1}$, respectively. On the contrary, the LES flows are driven by the constant background pressure gradient $\Delta P_{x}$ that ends up in a constant friction velocity $u_{\tau}=0.0469 \mathrm{~m} \mathrm{~s}^{-1}$ and a range of free-stream wind speed $0.555 \mathrm{~m} \mathrm{~s}^{-1} \leq U_{\infty} \leq 1.010 \mathrm{~m} \mathrm{~s}^{-1}$. Therefore, the friction factor $f$ in the wind tunnel experiments and the LES are ranged from $8 \times 10^{-3}$ to $13 \times 10^{-3}$ and $4.3 \times 10^{-3}$ to $14.3 \times 10^{-3}$, respectively. In the wind tunnel experiments, the friction factor is found peaked at $h / b=1 / 8$ with free-stream wind speed $U_{\infty}=6.7 \mathrm{~m} \mathrm{~s}^{-1}$ (higher wind speed). In the LES, on the other hand, the friction factor increases with decreasing $h / b$ that is peaked at the roughness element configuration of least $h / b(=1 / 11)$. The Reynolds number $\operatorname{Re}_{\delta}\left(=U_{\infty} \delta / v\right.$; where $v$ is the kinematic viscosity) measured in terms of TBL thickness $\delta$ and freestream mean wind speed $U_{\infty}$ is over 60,000 in both the wind tunnel experiments and the LES. It is noteworthy that, though the wind-tunnel measurements and LES results are tested at different free-stream wind speeds, their output can be compared in a dimensionless manner because the Reynolds number is over the critical value.

The ensemble-averaged mean-wind speeds $\langle\bar{u}\rangle / U_{\infty}$ over rough surfaces generally resemble the characteristic open-channel flows (Fig. 3a) that increases rapidly with increasing wall-normal distance $z$, converging gradually to its free-stream value $U_{\infty}$ at the top. The influence of rough surfaces on the dynamics is clearly depicted in the LES-calculated mean-wind speeds such that the wind shear in the near-wall region $(z \leq 0.1 \delta)$ decreases with increasing friction factor $f$. On the other hand, the ensemble-averaged mean-wind-speed profiles show only a tiny difference over different rough surfaces in the wind tunnel experiments. The less notable variation compared with their LES counterpart is mainly attributed to the nature of turbulence generation. In the LES (with infinitely large horizontal extent), the turbulence is generated solely by the mechanical shear induced by the bottom rough surfaces. Apart from the bottom rough surfaces, turbulence is unavoidably generated upstream (by the blower and the developing section) in the wind-tunnel experiments. As a result, the background
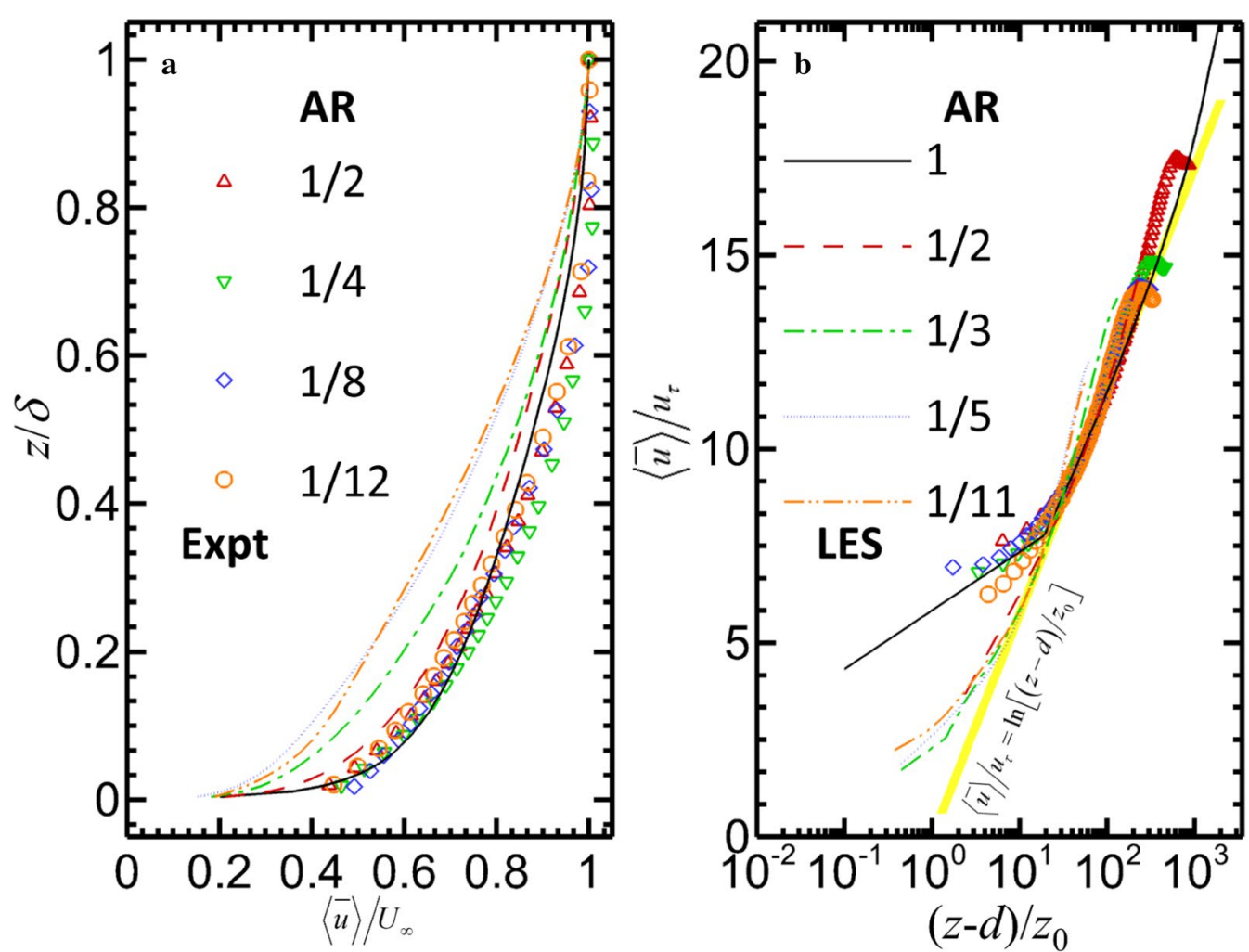

Fig. 3 Dimensionless mean-wind-speed $\langle\bar{u}\rangle$ profiles plotted as functions of wall-normal distance $z$ in: $\mathbf{a}$ linear and $\mathbf{b}$ logarithmic scales 
turbulence in the wind-tunnel test section is higher than that in the LES, resulting in a more uniform ensembleaveraged mean-wind-speed profiles over different rough surfaces.

Flows over rough surfaces can be expressed in the form of conventional logarithmic law of the wall (log-law)

$$
\frac{\langle\bar{u}\rangle}{u_{\tau}}=\frac{1}{\kappa} \ln \left(\frac{z-d}{z_{0}}\right)
$$

in the inertial sublayer (ISL) where $\kappa(=0.42)$ is the von Kármán constant, $d$ the displacement height and $z_{0}$ the roughness length over non-smooth surfaces. The log-law is clearly depicted in the current wind-tunnel experiments and LES (Fig. 3b). Whereas, it is noteworthy that the ensemble-averaged mean-wind-speed profiles deviate from the theoretical log-law while approaching the bottom rough surfaces in the roughness sublayer (RSL; Ho and Liu 2017). Hence, the flows are more uniform than those predicted by the log-law. The wind-tunnel measured mean-wind-speed profiles over the four roughsurface configurations show a similar behavior. The LEScalculated mean-wind speeds for unity AR follow the wind-tunnel measured ones closely as well. However, the
LES results from other settings exhibit a different behavior in which a deeper inertial sublayer (ISL) is observed. The dissimilar behavior is likely attributed to the different turbulence generation mechanism in the wind-tunnel experiments and the LES mentioned previously.

In view of the rough-surface generated turbulence, the fluctuating velocities are peaked in the near-wall region in both the wind-tunnel experiments and the LES (Fig. 4a). Both dimensionless streamwise $\left\langle u^{\prime \prime} u^{\prime \prime}\right\rangle^{1 / 2} / u_{\tau}$ and vertical $\left\langle w^{\prime \prime} w^{\prime \prime}\right\rangle^{1 / 2} / u_{\tau}$ fluctuating velocities are peaked in the near-wall region $(z \leq 0.1 \delta)$. The LES-calculated fluctuating velocities are essentially peaked in the near-wall region. It has highly refined spatial resolution close to the rough surfaces that calculated well the diminishing wind speeds right over the solid boundaries. The streamwise fluctuating velocity is peaked over the roughness elements that decreases with increasing wall-normal distance thereafter. The vertical fluctuating velocity, on the other hand, shows a relatively uniform level extending from the near-wall region to the TBL flow core. Its variation is less than $10 \%$ in the range $0 \leq z \leq 0.4 \delta$. Comparatively, the streamwise fluctuating velocity drops for
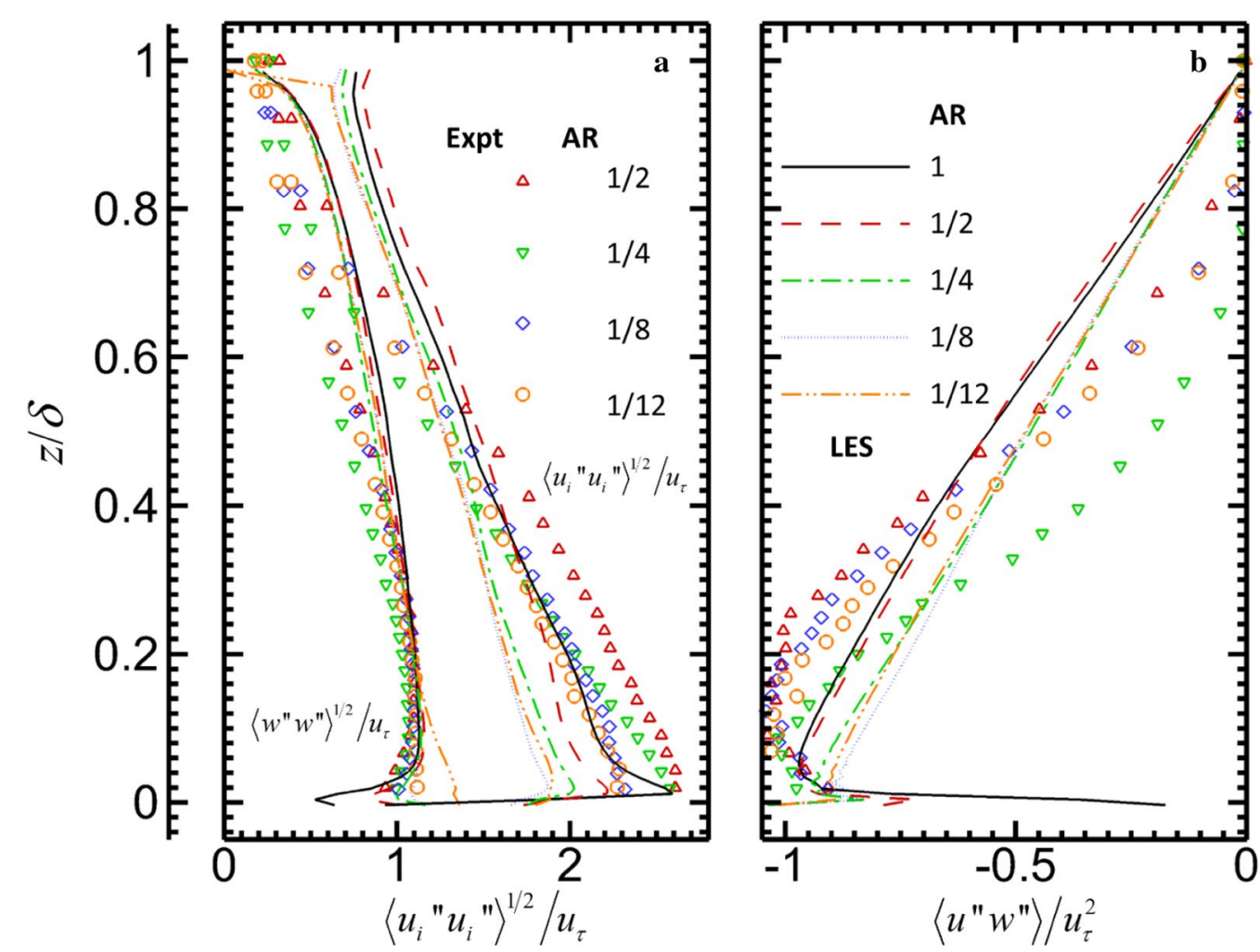

Fig. 4 Dimensionless profiles of: a fluctuating velocities $\left\langle u_{i}^{\prime \prime} u_{i}^{\prime \prime}\right\rangle^{1 / 2}$ and $\mathbf{b}$ turbulent momentum flux $\left\langle u^{\prime \prime} w^{\prime \prime}\right\rangle$ plotted as functions of wall-normal distance $z$ 
almost 50\%. Nonetheless, the fluctuating-velocity data from wind-tunnel experiments and LES agree well with each other.

The vertical momentum fluxes $\left\langle u^{\prime \prime} w^{\prime \prime}\right\rangle / u_{\tau}^{2}$ over idealized rough surfaces also exhibit the behavior alike their smooth-surface counterparts. The uniform-stress regions are observed in the flows over different rough surfaces in both wind-tunnel experiments and LES (Fig. 4b). However, the wind-tunnel results show a thicker uniform-stress region but the LES is (about 30\%) thinner. Hence, additional analyses will be conducted to look into this regard. Besides, the two solution approaches show different decreasing rates of momentum flux in the wall-normal direction. Comparatively, the wind-tunnelcalculated momentum fluxes decrease faster than the LES-calculated ones. The difference is likely attributed to the different definitions of TBL. In the wind-tunnel experiments, the TBL thickness is defined at the wallnormal distance where the ensemble-averaged meanwind speed converges to $99 \%$ of the free-stream value, i.e. $\langle\bar{u}(\delta)\rangle=0.99 U_{\infty}$. On the other hand, the TBL thickness is prescribed at the LES domain top because of the BCs employed in the mathematical models.

The plume dispersion behind a ground-level source over different rough surfaces, by and large, exhibits the theoretical Gaussian behavior as shown previously. The dimensionless tracer-concentration profiles from windtunnel measurements and LES agree reasonably well with the theoretical Gaussian profiles at different streamwise distance $x$ after the ground-level tracer source (Fig. 5). The LES-calculated tracer concentration over the rough surface of unity AR is slightly smaller than the Gaussian solution while the concentrations of other ARs follow closely which are in line with the theory. The wind-tunnel measured tracer concentrations, on the other hand, are slightly higher than those of the Gaussian solution. The discrepancy, from the instrumentation point of view, is caused by the non-zero effective emission height in the wind tunnel experiments. The tracer, which is represented by water vapor in the wind tunnel experiments, is driven from the water tank to the line source then emitted into the wind-tunnel test section by an electric fan. Under this circumstance, the non-zero discharge momentum at the ground-level line source induces initial plume rise that is estimated in the order of $h$ over the roughness elements approximately. The non-zero initial plume rise modifies the plume trajectory. It is no longer along the ground level that subsequently affects the calculation of vertical dispersion coefficient $\sigma_{z}$. The initial plume rise can also be explained by elevated maximum
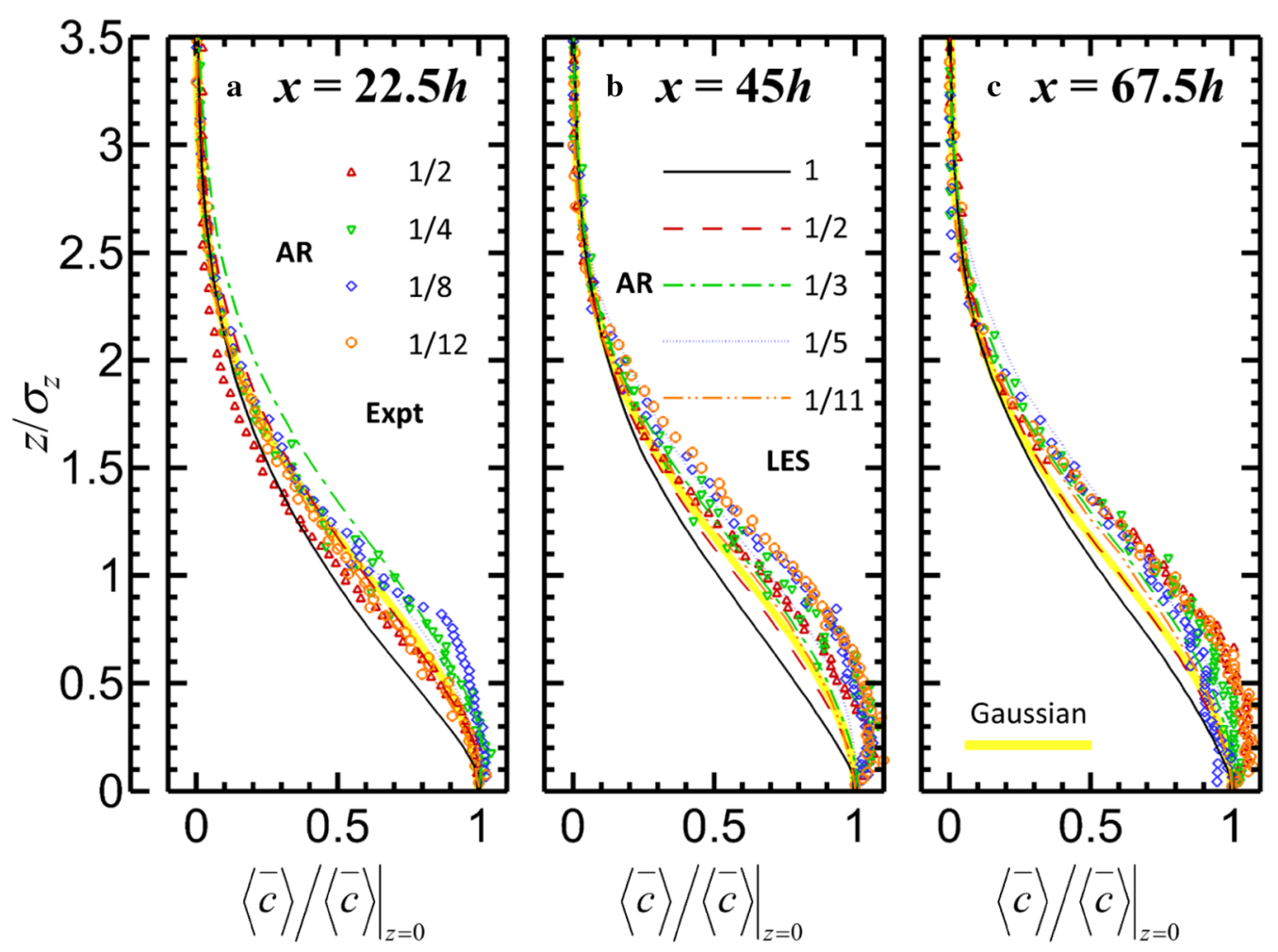

Fig. 5 Dimensionless tracer-concentration $\langle\bar{c}\rangle$ profiles plotted as functions of wall-normal distance $z$ at $x=: \mathbf{a} 22.5 \mathrm{~h} ; \mathbf{b} 45 \mathrm{~h}$ and $\mathbf{c} 67.5 \mathrm{~h}$ 
tracer concentration obtained in the wind tunnel experiments (approximately at $z=0.25 \sigma_{z}$ ), which, however, is not observed in the LES.

The tracer concentrations behave alike Gaussian shape in different streamwise distance, for instant, $x=22.5 \mathrm{~h}$ (Fig. 5a), 45h (Fig. 5b) and 67.5h (Fig. 5c). The theoretical Gaussian solution only applies to far-field plume dispersion where $\sigma_{z} \propto x^{1 / 2}$ so a notable discrepancy should be observed in the near field where $\sigma_{z} \propto x$. The streamwise location closest to the ground-level line source in the current wind tunnel experiments is at $x=22.5 \mathrm{~h}$. Nonetheless, both results of wind-tunnel experiments and LES follow well the theoretical (far-field) Gaussian-concentration profiles. It is noteworthy that surface roughness indeed shortens the near-field extent (Ng and Liu 2013). It in turn favors the applicability of the parameterization newly developed in this paper.

\section{Discussion}

The vertical dispersion coefficient $\sigma_{z}$ obtained from the wind-tunnel experiments and LES is plotted as a function of stream distance $x$, TBL thickness $\delta$ and drag coefficient $f$ alike the theoretical parameterization Eq. (4) in Fig. 6. The size of roughness elements $h$ is adopted as the characteristic length scale. Favorable agreement among wind-tunnel experiments, LES and theoretical parameterization is clearly observed such that the vertical dispersion coefficients $\sigma_{z}$ obtained from different approaches are proportional to the newly derived dimensionless parameter $(x / h)^{1 / 2} \times(\delta / h)^{1 / 2} \times f^{1 / 2}$ in a dimensionless manner. Whereas their $y$-intercepts are apparently different, therefore, further investigation is needed to improve the parameterization. Generally, the

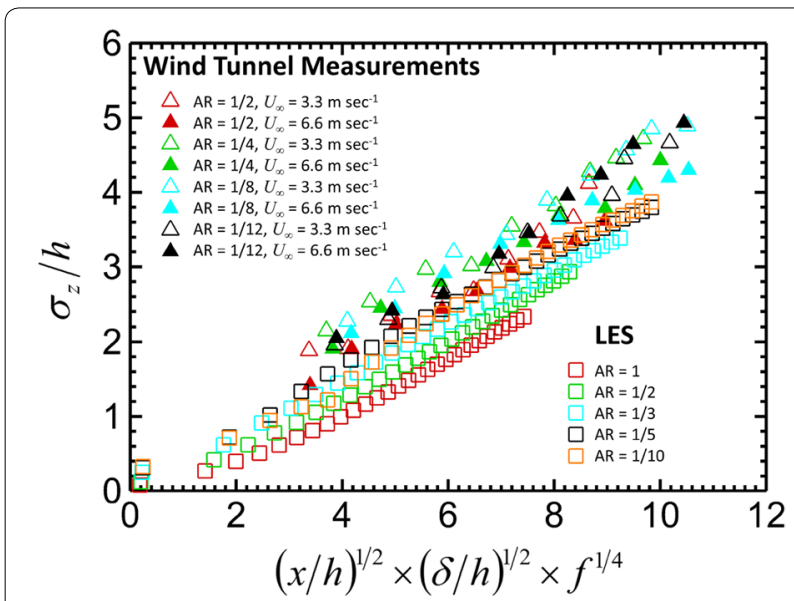

Fig. 6 Dimensionless profiles of vertical dispersion coefficient $\sigma_{z}$ plotted against streamwise distance $x$, TBL thickness $\delta$ and the friction factor $f$ wind-tunnel-measured vertical dispersion coefficients are higher than their LES-calculated counterparts that are likely attributed to the non-zero effective emission height and the associated elevated plume trajectory. Whereas, the plume trajectory in the LES is at the ground level exactly. The wind-tunnel results of vertical dispersion coefficients, though scattering, collapse reasonably well for different ARs. Apparently, they are independent from Reynolds number as shown by the datasets tested in different levels of mean-wind speeds. The molecular viscosity thus has minimal effect so the newly proposed parameterization should be applicable to other length scales as well. On the contrary, the LES-calculated vertical dispersion coefficients increase with increasing drag coefficient. Additional analyses are thus required to look into that regard to refine the parameterization. The ensemble-averaged mean-wind speeds for flows over different rough surfaces are rather uniform in the wind-tunnel experiments. Whereas notable differences are observed in the LES-calculated flows in view of the BCs and domain configuration. The dissimilar dynamic, which is attributed to the different turbulence generation mechanism, is likely the reason leading to the discrepancy between the concentrations obtained in the LES and the wind tunnel measurements.

\section{Conclusions}

Wind-tunnel experiments and LES are conducted concurrently to examine the plume dispersion over hypothetical urban areas in the form of identical ribs in crossflows. The tracer concentrations over arrays of street canyons of different ARs (aerodynamic resistance) exhibit the conventional Gaussian profiles of concentration. Along with the analytical derivation, an improved parameterization of pollutant plume dispersion coefficient is demonstrated such that $\sigma_{z} \propto x^{1 / 2} \times \delta^{1 / 2} \times f^{1 / 4}$. Unlike the conventional dispersion coefficient for Gaussian models. The newly proposed parameterization can be employed to estimate plume dispersion in response to the aerodynamic resistance over urban areas in isothermal conditions.

Although the results are favorable, we note that the current TBL thickness tested is rather shallow that would suppress the vertical plume dispersion. Besides, only ribs are tested in this paper that would limit the applicability of the newly developed parameterization for cubical roughness elements or even realistic urban morphology. Moreover, thermal stratification is not yet included in the analyses, therefore, the current findings are only applicable to the ABL bottom in the vicinity of buildings. The rather shallow TBL weakens the performance of parameterization that overlooks the turbulent transport processes driven by atmospheric 
stratification. Generally, unstable and stable stratification, respectively, enhances and weakens the transport processes. Additional studies, such as roughness elements of different sizes, will be performed to address the limitation of the current work as well as to improve the parameterization.

\begin{abstract}
Abbreviations
2D: two-dimensional; AR: aspect ratio ( $=h / b)$; BC: boundary condition; CFD: computational fluid dynamics; $C$ T: constant-temperature; DESA: Department of Economic and Social Affairs, United Nations; FVM: finite volume method; HWA: hot-wire anemometer; ISL: inertial sublayer; ISVR: Institute of Sound and Vibration; Log-law: logarithmic law of the wall; NI: National Instruments; LAN: local area network; LES: large-eddy simulation; PBiCG: pre-conditioned bi-conjugate gradient method; PCG: pre-conditioned conjugate gradient method; PISO: pressure-implicit with splitting of operators; RSL: roughness sublayer; SGS: subgrid-scale; SIMPLE: semi-implicit method for pressure linked equations; TBL: turbulent boundary layer; TKE: turbulence kinetic energy; WHO: World Health Organization; $b$ : separation between roughness elements; $d$ : displacement height; f: friction factor $\left(=2 U_{T}^{2} / U_{\infty}^{2}\right) ; h$ : size of roughness elements; $k_{z}$ : eddy diffusivity in the vertical direction; $L_{i}$ number of cells in the $\zeta$ direction; $N_{\zeta}$. number of cells in the $\zeta$ direction; $R_{\delta}$ : Reynolds number $\left(=U_{\infty} \delta / v\right) ; t$ : pollutant travel time; $U$ : mean-wind speed; $U_{\infty}$ : free-stream mean wind speed; $u$ : streamwise velocity component; $u_{\tau}$ : friction velocity; $w$ : vertical velocity component; $x$ : distance after the pollutant source in the streamwise direction; $z$ : wall-normal distance in the vertical direction; $z_{0}$ : roughness length scale; $\Delta P_{x}$ : background pressure gradient; $\delta$ : turbulent boundary layer thickness; $k$ : von Kármán constant $(=0.42)$; $v$ : kinematic viscosity; $\sigma_{z}$ : vertical dispersion coefficient; $\langle\bar{\varphi}\rangle$ : ensemble average of variable $\varphi ; \varphi^{\prime \prime}$ : fluctuating component of variable $\varphi(=\varphi-\langle\bar{\varphi}\rangle$ ).
\end{abstract}

\section{Authors' contributions}

C-HL conducted the analysis and drafted the manuscript. ZM carried out the wind tunnel experiments. ZW performed the LES. All authors read and approved the final manuscript.

\section{Acknowledgements}

Zhangquan Wu thanks the Hong Kong (HK) Research Grants Council (RGC) for the financial support through the Hong Kong Ph.D. Fellowship (HKPF) scheme. This research is conducted in part using the research computing facilities and/ or advisory services offered by Information Technology Services (ITS), The University of Hong Kong (HKU). Technical support from Ms. Lilian Y.L. Chan, Mr. W.K. Kwan and Mr. Bill H.T. Yau is appreciated. This study is partly supported by the General Research Fund (GRF) 17205314 of HK RGC.

\section{Competing interests}

The authors declare that they have no competing interests.

\section{Availability of data and materials}

Not applicable.

\section{Consent for publication}

Not applicable

\section{Ethics approval and consent to participate}

Not applicable.

\section{Funding}

Hong Kong Ph.D. Fellowship (HKPF) scheme and General Research Fund (GRF) 17205314 of HKRGC

\section{Publisher's Note}

Springer Nature remains neutral with regard to jurisdictional claims in published maps and institutional affiliations.
Received: 22 February 2018 Accepted: 31 August 2018

Published online: 22 September 2018

\section{References}

Briant R, Seigneur C, Gadrat M, Bugajny C (2013) Evaluation of roadway Gaussian plume models with large-scale measurement campaigns. Geosci Model Dev 6:445-456

Briggs GA (1973) Diffusion estimation for small emissions. Atmospheric turbulence and diffusion laboratory contribution. 79

Britter RE, Hanna SR (2003) Flow and dispersion in urban areas. Annu Rev Fluid Mech 35:469-496

Bruun H (1971) Interpretation of a hot wire signal using a universal calibration law. J Phys E 4:225

Cheng H, Castro IP (2002) Near wall flow over urban-like roughness. Boundary Layer Meteorol. 104:229-259

Chung J, Hagishima A, Ikegaya N, Tanimoto J (2015) Wind-tunnel study of scalar transfer phenomena for surfaces of block arrays and smooth walls with dry patches. Boundary Layer Meteorol. 157:219-236

Davidson MJ, Mylne KR, Jones CD, Phillips JC, Perkins RJ, Fung JCH, Hunt JCR (1995) Plume dispersion through large groups of obstacles—a field investigation. Atmos Environ 29:3245-3256

Desa A (2016) The world's Cities in 2016, United Nations. DESA, New York. https ://doi.org/10.18356/8519891f-en

Gromke C (2011) A vegetation modeling concept for building and environmental aerodynamics wind tunnel tests and its application in pollutant dispersion studies. Environ Pollut 159:2094-2099

Ho Y-K, Liu C-H (2017) A wind tunnel study of flows over idealised urban surfaces with roughness sublayer corrections. Theor Appl Climatol 30:305-320

Inagaki A, Castillo MCL, Yamashita Y, Kanda M, Takimoto H (2012) Large-eddy simulation of coherent flow structures within a cubical canopy. Boundary Layer Meteorol. 142:207-222

Lelieveld J, Evans JS, Fnais M, Giannadaki D, Pozzer A (2015) The contribution of outdoor air pollution sources to premature mortality on a global scale. Nature 525:367-371

MacDonald RW, Griffiths RF, Hall DJ (1998) A comparison of results from scaled field and wind tunnel modelling of dispersion in arrays of obstacles. Atmos Environ 32:3845-3862

Mavroidis I, Griffiths RF (2001) Local characteristics of atmospheric dispersion within building arrays. Atmos Environ 35:2941-2954

Mo Z, Liu C-H (2017) On the turbulence structure over different surface roughness: a perspective from wind tunnel measurements. In: Urban meteorology and climate conference, City University of Hong Kong, Hong Kong, 25-26 May 2017

Moreira DM, Vilhena MT, Tirabassi T, Costa C, Bodmann B (2006) Simulation of pollutant dispersion in the atmosphere by the Laplace transform: the ADMM approach. Water Air Soil Poll. 177:411-439

$\mathrm{Ng}$ C-T, Liu C-H (2013) Parameterization of pollutant plume dispersion in neutral stratification over hypothetical urban areas. In: 13th EMS annual meeting \& 11 th European conference on applications of meteorology (ECAM), Reading, UK, 9-13 September

Oke TR (1988)'Street design and urban canopy layer climate. Energy Build 11:103-113

OpenFOAM (2018) OpenFOAM: The OpenFOAM Foundation, http://www. openfoam.org/. Accessed 16 May 2018

Pasquill F, Smith FB (1983) Atmospheric diffusion, 3rd edn. Wiley, New York

Raupach MR, Antonia RA, Rajagopalan S (1991) Rough-wall turbulent boundary layers. Appl Mech Rev 44:1-25

Roberts OFT (1923) The theoretical scattering of smoke in a turbulent atmosphere. Proc Roy Soc Lond A 104:640-654

Scheffe RD, Strum M, Phillips SB, Thurman J, Eyth A, Fudge S, Morris M, Palma T, Cook R (2016) Hybrid modeling approach to estimate exposures of hazardous air pollutants (HAPS) for the national air toxics assessment (NATA). Environ Sci Technol 50:12356-12364

Schumann U (1975) Subgrid scale model for finite difference simulations of turbulent flows in plane channels and annuli. J Comp Phys 18:376-404

Sharma V, Myrup OL (1975) Diffusion from a line source in an urban atmosphere. Atmos Environ 9:907-922 
Skupniewicz CE, Schacher GE (1986) Parameterization of plume dispersion over water. Atmos Environ 20:1333-1340

Smagorinsky J (1963) General circulation experiments with the primitive equations I: the basic experiment. Month Weath Rev 91:99-165

Tsuang B-J, Lee C-T, Cheng M-T, Lin N-H, Lin Y-C, Chen C-L, Peng C-M, Kuo P-H (2003) Quantification on the source/receptor relationship of primary pollutants and secondary aerosols by a Gaussian plume trajectory model: part III-Asian dust-storm period. Atmos Environ 37:4007-4017

Turner DB (1994) Workbook of atmospheric dispersion estimates: an introduction to dispersion modeling. Lewis Publishers, London

Venkatram A, Isakov V, Pankratz D, Yuan J (2005) Relating plume spread to meteorology in urban areas. Atmos Environ 39:371-380
Walcek CJ (2002) Effect of wind shear on pollution dispersion. Atmos Environ 36:511-517

WHO (2016) WHO's Urban Ambient Air Pollution Database_-Update 2016, Public Health, Social and Environmental Determinants of Health Department, World Health Organization, 1211 Geneva 27, Switzerland. http://www. who.int/phe. Accessed 16 May 2018

Wong CCC, Liu C-H (2013) Pollutant plume dispersion in the atmospheric boundary layer over idealized urban roughness. Bound Layer Meteor. 147:281-300

Wu Z, Liu C-H (2017) Budget analysis for reactive plume transport over urban roughness. In: Urban meteorology and climate conference, City University of Hong Kong, Hong Kong, 25-26 May 2017

\section{Submit your manuscript to a SpringerOpen ${ }^{\circ}$ journal and benefit from:}

- Convenient online submission

- Rigorous peer review

- Open access: articles freely available online

- High visibility within the field

- Retaining the copyright to your article

Submit your next manuscript at $\gg$ springeropen.com 